\title{
PERSPECTIVE
}

\section{SHARING THE MESSAge}

One of CIF/IFC's communication goals is to inform its membership and other interest groups about Canadian forestry issues and practices. In addition to this, the membership through their work, regularly work to raise the awareness of the importance of forestry economically and socially in Cana$\mathrm{da}$, as well as to promote through professional practice the role of the forest practitioner in forest management.

One of the communication vehicles for the $\mathrm{CIF} / \mathrm{IFC}$ is The Forestry Chronicle. It provides professional, technological and scientific information to aid forest practitioners in conducting sound forest management practices. It also reports on section activities, e.g., field tours and technical sessions, and on university and technical school news. The Forestry Chronicle is distributed across Canada through its membership, and also worldwide through subscribers. The most important communication network that the $\mathrm{CIF} / \mathrm{IFC}$ has is through its sections and membership. Through their own work within government, industry, communities, and other sectors members perform the important task of facilitating public awareness of the practice of forestry. Through this knowledge the public can then influence decision-makers by identifying forestry resource issues and values. Equally important, our members network to continually keep each other informed of the latest research and developments in forestry, and across the sectors.

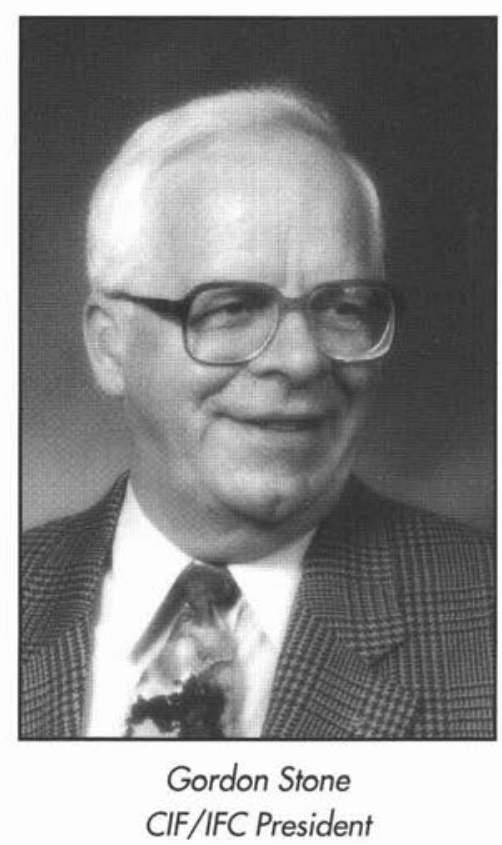

A key message that we should all be delivering, especially with the competing demands on youth to follow careers in the high tech industry, is to communicate that forest practitioners are a highly skilled, professional, knowledge workforce. We are leaders in our practice. We are no longer hewers of wood. Forest practitioners are the human face for forestry.

Communication is an ever-present opportunity each and every day of life, at work and in public to get the CIF/IFC message across to Canadians and the international community. We must make the most of each opportunity, and work together to keep Canada as a leader in forestry and the message that forest practitioners are a proud and strong knowledge workforce.

Gordon L. Stone President, CIF/IFC

\section{LA DIFFUSION DU MESSAGE}

L'un des objectifs de l'Institut au niveau des communications est d'informer ses membres et les autres groupes d'intérêts au sujet des enjeux et des pratiques de foresterie au canada. En plus de cela, les membres par l'entremise de leur travail, cherche régulièrement à accroître la sensibilisation en ce que a trait à l'importance de la foresterie au niveau économique et social au Canada, en plus de promouvoir par leur pratique professionnelle le rôle de praticien forestier en aménagement forestier.

L'un des véhicules de communication de l'Institut consiste dans Le Forestry Chronicle. Cette revue apporte de l'information professionnelle, technologique et scientifique pour aider les praticiens forestiers à entreprendre de saines pratiques d'aménagement forestier. Elle contient également des articles sur les activités des sections, p. ex. les excursions techniques, les sessions techniques; ainsi que des nouvelles des universités et des établissements techniques. Le Forestry Chronicle est distribué partout au Canada par l'entremise de ses membres, et également dans le monde entier grâce à ses abonnés. Le plus important réseau de communication sur lequel 'Institut peut compter réside dans ses sections et ses membres. Par l'entremise de leur propre travail au sein des gouvernements, de l'industrie, des collectivités et autres secteurs, les membres accomplissent l'importante tâche de faciliter la sensibilisation du public en matière de pratique de la foresterie. Grâce à cela, le public peut ainsi influencer les preneurs de décision en identifiant les enjeux et les valeurs rattachés aux ressources forestières. Le réseau de nos membres nous permet d'une façon toute aussi importante de demeurer constamment informé des derniers développements en matière de recherche et en foresterie et ce pour tous les secteurs. Le message-clé que nous devrions tous livrer, tout spécialement dans un contexte de compétition pour les jeunes qui veulent suivre une carrière dans l'industrie de haute technologie, est de communiquer que les praticiens de la foresterie constituent une main d'oeuvre hautement qualifiée, professionnelle et bien instruite. Nous sommes les chefs de file dans notre pratique. Nous ne sommes plus des coupeurs de bois. Les praticiens forestiers constituent le visage humain de la foresterie.

Il faut saisir toute opportunité chaque jour et tous les jours, au travail et en public, de transmettre le message de l'Institut à tous les Canadiens et à la communauté internationale. Nous devons tirer partie de chacune des opportunités, et nous devons travailler ensemble pour faire en sorte que le Canada demeure un chef de file en foresterie et que le message que les praticiens forestiers constituent une main d'oeuvre fière et bien instruite soit diffusé.

Gordon L. Stone, Président, CIF/IFC 